\title{
Science Case for the LAMA Telescope
}

\author{
Kenneth M. Lanzetta ${ }^{a}$ and Paul Hickson ${ }^{b}$ \\ ${ }^{a}$ Stony Brook University, Department of Physics and Astronomy, Stony Brook, NY \\ 11794-3800, U.S.A. \\ ${ }^{b}$ University of British Columbia, Department of Physics and Astronomy, 6224 Agricultural \\ Road, Vancouver, BC V6T 1Z1, CANADA
}

\begin{abstract}
Aspects of the science case for the Large-Aperture Mirror Array or LAMA telescope are presented. The LAMA telescope will be a large-aperture array of 66 6.15-m diameter primary mirrors operating together to give a lightcollecting ability and angular resolution comparable to those of a conventional 50-m diameter telescope. The first-generation instruments of the LAMA telescope will include a multi-band optical- and infrared-wavelength camera and a high-dispersion echelle spectrograph. The most important difference between the LAMA telescope and conventional telescopes is that the LAMA telescope will be constrained to point and track within 4 deg of the zenith, which corresponds to a maximum tracking time per night of $\approx 30 \mathrm{~min}$. This implies that deep observations obtained by the LAMA telescope will be obtained gradually-over periods spanning weeks, months, or years - and so will contain a temporal dimension as a natural consequence of the design and mode of operation of the telescope. This temporal dimension makes possible some of the most interesting science to be performed with the LAMA telescope, ranging from the identification of very high redshift supernovae through observation of the acceleration of the Ly $\alpha$-forest absorption systems as a direct probe of the expansion history of the universe. The LAMA telescope will be used to carry out an extremely deep, narrow-field imaging survey at optical and infrared wavelengths and a high spectral resolution spectroscopic survey of bright, high-redshift QSOs.
\end{abstract}

Keywords: Cosmology, Extra-solar planets, Galaxy formation, Galaxy evolution, QSO absorption lines

\section{INTRODUCTION}

The Large-Aperture Mirror Array or LAMA telescope will be a large-aperture array of 66 6.15-m diameter primary mirrors operating together to give a light-collecting ability and angular resolution comparable to those of a conventional 50-m diameter telescope. The LAMA telescope will be used to address important issues in cosmology and extragalactic and galactic astronomy, including issues related to faint galaxies and supernovae, QSO absorption lines, faint stars, and extra-solar planets.

The LAMA telescope will differ from conventional telescopes in three important ways:

1. Near-zenith pointing and tracking: The telescope will be constrained to point and track within 4 deg of the zenith.

2. Distributed-aperture configuration: The telescope will be comprised of a number of moderate-aperture telescopes working together to give the performance of a single large-aperture telescope.

3. Liquid-mercury primary mirrors: The telescope will employ liquid-mercury primary mirrors, which can be built at a cost of less than $5 \%$ of the cost of comparable glass primary mirrors.

The first of these differences - namely the constraint of near-zenith pointing and tracking-has very important implications for the scientific program of the LAMA telescope, both positive and negative. A primary objective of the present work is to examine the issues and tradeoffs associated with this crucial design decision. A secondary objective of the present work is to describe some of the science objectives of the LAMA telescope project, with particular emphasis on topics related to the scientific interests and background of the authors.

The most important implication of the constraint of near-zenith pointing and tracking of the LAMA telescope is that deep observations will be obtained gradually, over periods spanning weeks, months, or years. This means 
that all deep observations obtained using the LAMA telescope will contain a temporal dimension as a natural consequence of the design and mode of operation of the telescope. This temporal dimension has been hitherto more or less neglected in deep observations by large-aperture telescopes and finds its closest analogues only in the very recent deep GOODS and UDF observations of faint galaxies and supernovae obtained by the Hubble Space Telescope. Yet this temporal dimension makes possible some of the most interesting science to be performed with the LAMA telescope, and we consider it to be one of the important benefits of the telescope.

The organization of the present work is as follows: The configuration, modes of operation, and performance of the telescope are described in $\S 2$, and the implications of the limited pointing and tracking abilities of the telescope are described in $\S 3$. Aspects of the science objectives of the project are described in $\S 4$. A brief summary and conclusion are presented in $\S 5$.

\section{CONFIGURATION, MODES OF OPERATION, AND PERFORMANCE}

The LAMA telescope will differ from other large-aperture telescopes, in both the way it will be designed and the way it will be used. In this section, we discuss the configuration, modes of operation, and performance of the LAMA telescope.

\subsection{Configuration}

\subsubsection{Pointing, Tracking, and Field of View}

The LAMA telescope will be constrained to point and track within $4 \mathrm{deg}$ of zenith. At a typical latitude (say near $\pm 30 \mathrm{deg}$ ), this will correspond to a maximum tracking time per night of $\approx 30 \mathrm{~min}$. The accessible field of view of the LAMA telescope (i.e. set by the pointing constraint) will be $\approx 6 \%$ of the entire sky, or $\approx 2475$ $\mathrm{deg}^{2}$. The instantaneous field of view of the LAMA telescope (i.e. set by the optical design and adaptive optics correction) will be $\approx 30$ arcsec in diameter.

\subsubsection{Incoherent and Coherent Optical Beam Combination}

The LAMA telescope will combine optical beams from the individual array elements either incoherently, to yield the light-collecting ability of a 50-m diameter telescope and the angular resolution of a 6.15-m diameter telescope, or coherently, to yield the light-collecting ability and angular resolution of a 50-m diameter telescope.

\subsubsection{First-Generation Instruments}

The LAMA telescope will be equipped with two first-generation instruments: a multi-band optical- and infraredwavelength camera and a high-dispersion echelle spectrograph. The multi-band camera will cover three to five optical- and three infrared-wavelength bands. Detectors of the camera will sample the instantaneous field of view (or at least some portion of the instantaneous field of view) at the Nyquist rate or greater. The high-dispersion spectrograph will cover optical wavelengths at a spectral resolution of $R \approx 100,000$.

\subsection{Modes of Operation}

\subsubsection{Imaging Mode of Operation}

In the imaging mode of operation, the LAMA telescope will combine the optical beams of the individual array elements either incoherently or coherently. In both the incoherent and coherent imaging modes of operation, the instantaneous field of view will be centered on a suitable phase reference source, which will provide phase reference for pointing, tip/tilt and higher-order adaptive optics correction, and (for the coherent mode of operation) coherent beam combination. The phase reference sources will be acquired up to $\approx 15$ min before transit and tracked until up to $\approx 15 \mathrm{~min}$ after transit. Long exposures will be obtained gradually, by repeatedly observing the same fields for up to $\approx 30$ min per night, over periods spanning weeks, months, or years. 


\subsubsection{Spectroscopic Mode of Operation}

In the spectroscopic mode of operation, the LAMA telescope will transfer the optical beams of the individual array elements to a series of high-dispersion echelle spectrograph elements. Each spectrograph element will accept the optical beams of several array elements through optical fiber feeds. In the spectroscopic mode of operation, the instantaneous field of view will be centered on the spectroscopic targets, which will serve as phase reference sources to provide phase reference for pointing and low-order adaptive optics correction only. The spectroscopic mode of operation will target only bright (i.e. brighter than they sky) point sources, so there is no particular advantage to incorporating higher-order adaptive optics correction or coherent beam combination. (This is true only for the first-generation high-dispersion echelle spectrograph; subsequent spectrographs may target other sources.) The spectroscopic targets will be acquired up to $\approx 15 \mathrm{~min}$ before transit and tracked until up to $\approx 15$ min after transit. Long exposures will be obtained gradually, by repeatedly observing the same targets for up to $\approx 30$ min per night, over periods spanning weeks, months, or years.

\subsection{Performance}

The performance of the LAMA telescope will be comparable to the performance of other large-aperture telescopes and will be set by the combined apertures of the individual array elements, the reflectivities of the mercury primary mirrors and subsequent optical surfaces, the quantum efficiencies of the detectors, and (for backgroundlimited observations) the image quality of the telescope, resulting from the performance of the adaptive optics correction and the coherent beam combination. The expected performance of the LAMA telescope is summarized in Table 1.

Table 1

Performance of LAMA Telescope

\begin{tabular}{|c|c|c|c|c|}
\hline \multirow[b]{3}{*}{ Exposure } & \multicolumn{4}{|c|}{$\begin{array}{c}5 \sigma \text { Point Source } \\
\text { Detection Threshold }\end{array}$} \\
\hline & \multicolumn{2}{|c|}{ Incoherent } & \multicolumn{2}{|c|}{ Coherent } \\
\hline & $f_{\nu}(\mathrm{pJy})$ & $A B$ & $f_{\nu}(\mathrm{pJy})$ & $A B$ \\
\hline$I$ band, $30 \mathrm{~min}$ & 645 & 31.9 & 103 & 33.9 \\
\hline$I$ band, $1500 \mathrm{~min}$ & 91 & 34.0 & 15 & 36.0 \\
\hline$K$ band, $30 \mathrm{~min}$ & 4750 & 29.7 & 606 & 31.9 \\
\hline$K$ band, $1500 \min \ldots \ldots \ldots \ldots$ & 672 & 31.8 & 86 & 34.1 \\
\hline
\end{tabular}

\section{IMPLICATIONS OF LIMITED POINTING AND TRACKING ABILITIES}

The scientific capabilities of the LAMA telescope will differ from the scientific capabilities of other large-aperture telescopes, due primarily to the condition that the telescope will be constrained to point and track within 4 deg of the zenith centered on a phase reference source. Aspects of these differences bear both positively and negatively on the scientific capabilities of the telescope. In this section, we discuss the implications of the limited pointing and tracking abilities of the LAMA telescope.

\subsection{Temporal Sequencing of Deep Observations}

Because it will be constrained to point and track within 4 deg of the zenith, the LAMA telescope will be limited to a maximum possible exposure time of $\approx 30 \mathrm{~min}$ per night. This means that long-exposure observations will be obtained over long periods of time, which implies that the method of operation of the LAMA telescope of necessity provides temporal sequencing of deep observations.

Consider observations of deep sources, i.e. sources which require long-exposure observations. Examples would be faint galaxies that require long-exposure observations to achieve detection or bright QSOs that require longexposure observations to achieve high signal-to-noise ratios. All long-exposure observations obtained by the LAMA telescope will be obtained gradually, over periods spanning weeks, months, or years. At the end of the relevant period, the observations could be summed to yield the equivalent of a single long-exposure observation. But at the end of the relevant period, the observations could also be treated as a temporal sequence, providing 
information about the time history of the sources under study. This means that all deep observations obtained by the LAMA telescope will contain a temporal dimension as a natural consequence of the design and mode of operation of the telescope. While other conventional telescopes could operate in a similar method and obtain similar observations, this then would obviate the large zenith-angle pointing and tracking abilities of conventional telescopes, and it would further run counter to the way conventional telescopes are scheduled.

This temporal dimension has been hitherto more or less neglected in deep observations by large-aperture telescopes and finds its closest analogues only in the very recent deep GOODS and UDF observations of faint galaxies and supernovae obtained by the Hubble Space Telescope. Yet this temporal dimension makes possible some of the most interesting science to be performed with the LAMA telescope-ranging from the identification of very high redshift supernovae through observation of the acceleration of the Ly $\alpha$-forest absorption systems as a direct probe of the expansion history of the universe-and we consider it one of the important benefits of the telescope.

\subsection{Scientifically Accessible Field of View}

Because it will be constrained to point and track within 4 deg of the zenith, the LAMA telescope will have an accessible field of view of $\approx 6 \%$ of the entire sky, or $\approx 2475 \mathrm{deg}^{2}$. But because it will also be constrained to point and track centered on a phase reference source, only some portion of the accessible field of view is relevant to the science objectives of the telescope. This "scientifically accessible field of view" will be smaller than the accessible field of view and will in general depend on the nature of the sources under consideration.

First consider observations of untargeted sources, i.e. sources for which the instantaneous field of view will be centered on a suitable phase reference source. Examples would be faint galaxies or supernovae or faint stars. Here the scientifically accessible field of view will be set by (1) the availability of suitable phase references sources and (2) the instantaneous field of view of the telescope. To provide phase reference for pointing, tip/tilt and higher-order adaptive optics correction, and coherent beam combination will probably require phase reference sources brighter than $V \approx 12$ to 15 . (The exact value will depend on the degree of correction required and on the detailed design of the telescope.) The availability of suitable phase reference sources is summarized in Table 2 , which lists the surface density of stars ${ }^{1}$ the number of stars in the accessible field of view, and the solid angle within the instantaneous field of view (i.e. within 30 arcsec in diameter) of stars in the accessible field of view versus limiting magnitude, for limiting magnitude in the range $V=12$ to 15 . (The values listed in Table 2 apply at high Galactic latitudes and hence represent lower limits to the actual values expected across the sky.)

Table 2

Availability of Suitable Phase Reference Sources

\begin{tabular}{|c|c|c|c|}
\hline$V$ & $\begin{array}{c}\text { Surface } \\
\text { Density }\left(\operatorname{deg}^{-2}\right)\end{array}$ & $\begin{array}{c}\text { Number } \\
\text { Accessible } \\
\end{array}$ & $\begin{array}{c}\text { Solid Angle } \\
\text { Accessible }\left(\mathrm{deg}^{2}\right)\end{array}$ \\
\hline 12 & 13 & 31,700 & 1.73 \\
\hline 13 & 27 & 64,800 & 3.53 \\
\hline 14 & 57 & 160,800 & 8.77 \\
\hline $15 \ldots \ldots \ldots \ldots \ldots \ldots$ & 135 & 324,000 & 17.67 \\
\hline
\end{tabular}

According to results of Table 2, many $\mathrm{deg}^{2}$ of the sky are accessible to the LAMA telescope for observations of untargeted sources. For subsequent follow-up observations (say with second-generation instruments), all sources identified using the first-generation instruments will be guaranteed to be within range of a suitable phase reference source.

Next consider observations of targeted sources, i.e. sources for which the source itself will serve as the phase reference source. Examples would be bright QSOs or bright nearby stars. Here the scientifically accessible field of view is identical to the accessible field of view, and the issue is simply whether sufficient examples of the class of sources under consideration can be found in the accessible field of view of the LAMA telescope. As a specific example, consider bright QSOs. The availability of QSOs is summarized in Table 3, which lists the surface density of $\mathrm{QSOs}^{2}$ and the number of QSOs in the accessible field of view versus limiting magnitude, for limiting magnitude in the range $B=16$ to 21 . 
Table 3

Availability of QSOs

\begin{tabular}{|c|c|c|c|}
\hline & $B$ & $\begin{array}{c}\text { Surface } \\
\text { Density }\left(\operatorname{deg}^{-2}\right)\end{array}$ & $\begin{array}{c}\text { Number } \\
\text { Accessible }\end{array}$ \\
\hline 16 & $\ldots \ldots$ & 0.0065 & 16 \\
\hline 17 & . & 0.084 & 208 \\
\hline 18 & . & 0.73 & 1808 \\
\hline 19 & $\ldots \ldots \ldots \ldots \ldots \ldots \ldots$ & 3.4 & 8464 \\
\hline 20 & $\ldots \ldots$ & 11 & 27,520 \\
\hline 21 & $\ldots \ldots \ldots \ldots \ldots \ldots$ & 27 & 66,240 \\
\hline
\end{tabular}

According to results of Table 3, tens of thousands of bright QSOs are accessible to the LAMA telescope. Some fraction of these QSOs will be of high redshift, i.e. suitable targets for absorption-line spectroscopy.

\subsection{Sensitivity of Observations}

Because it will be constrained to point and track within 4 deg of the zenith, the LAMA telescope will have access to fewer sources than conventional telescopes will have access too. For observations of common, faint sources, this will have no bearing on the scientific capabilities of the telescope. But for observations of rare, bright sources, the sources that will be observed by the LAMA telescope will be on average fainter than the sources that will be observed by conventional telescopes, which will effectively decrease the "sensitivity" of the LAMA telescope in comparison to conventional telescopes.

First consider observations of the faintest instances of a class. Examples would be the faintest accessible high-redshift galaxies and supernovae or the faintest accessible stars. Here an observational program will proceed by targeting sources in more or less random regions of the sky, and the sources that will be observed by the LAMA telescope will be on average identical to the sources that will be observed by conventional telescopes.

Next consider observations of the brightest instances of a class. Examples would be the brightest high-redshift QSOs or the brightest nearby stars that harbor extrasolar planets. Here an observational program would proceed by targeting the brightest $N$ accessible examples of the class (in order, say, to collect as much light as possible in the shortest exposure time possible), where $N$ is set to satisfy the specific science objectives of the program. Because the LAMA telescope will have access to fewer sources than conventional telescopes, the sources that will be observed by the LAMA telescope will be on average fainter than the sources that will be observed by conventional telescopes. The amount by which the sources that will be observed by the LAMA telescope will be fainter than the sources that will be observed by conventional telescope will depend on (1) the ratio of the solid angle of the sky accessible to the LAMA telescope to the solid angle of the sky accessible to the conventional telescope and (2) the shape of the number versus magnitude relation $n(m)$ of the sources.

Assume that the solid angle of the sky accessible to the LAMA telescope is $\Omega_{\text {LAMA }}$ and that the solid angle of the sky accessible to the conventional telescope is $\Omega_{\text {other }}$. Further assume that the number versus magnitude relation $n(m)$ of the sources has the form

$$
\log n(m)=\beta m+\text { const, }
$$

where $m$ is the magnitude of the source, and $n(m)$ is the surface density of the sources brighter than $m$, and $\beta$ characterizes the shape of the relation. If $N$ sources must be observed to satisfy the specific science objectives of the program, then the magnitude of the faintest source that must be observed is set by equating $n(m) \Omega=N$, which from equation (1) yields

$$
m=\frac{1}{\beta}(\log N-\log \Omega-\text { const }) .
$$

Evaluating equation (2) for both the LAMA telescope and the conventional telescope and subtracting yields the magnitude difference between the faintest source that must be observed by the LAMA telescope and the faintest source that must be observed by the conventional telescope of

$$
\Delta m=\frac{1}{\beta} \log \frac{\Omega_{\text {LAMA }}}{\Omega_{\text {other }}} .
$$




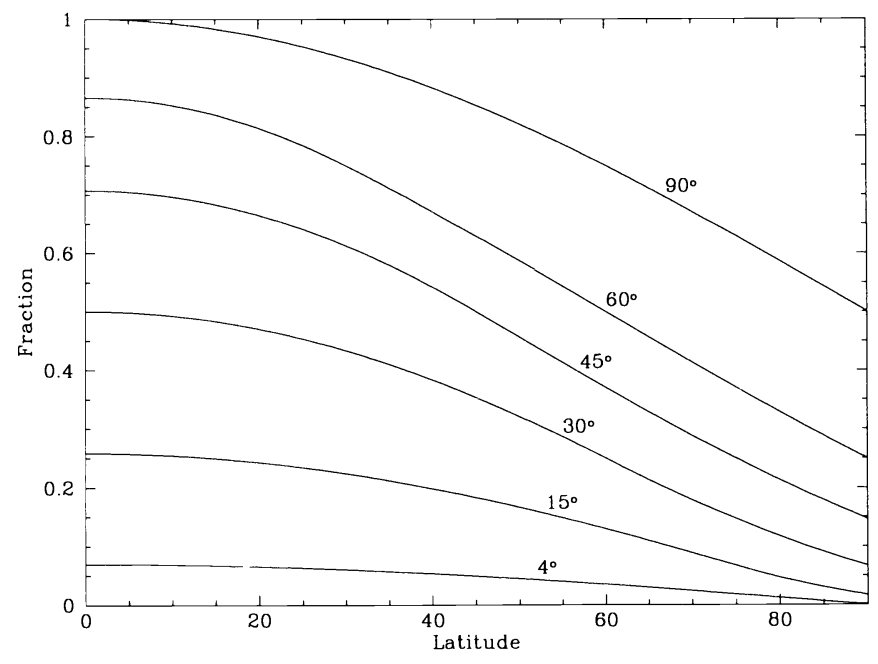

Figure 1. Fraction of the sky accessible, as functions of latitude and maximum zenith angle, for maximum zenith angles of $4,15,30,45$, and $90 \mathrm{deg}$.

In other words, for observations of the brightest instances of a class, the sources observed by the LAMA telescope will be fainter than the sources observed by a conventional telescope by an amount given by equation (3).

The number versus magnitude relation $n(m)$ of stars $^{1}$ is characterized by $\beta \approx 0.7$ at $B \lesssim 20$ and $\beta \approx 0.3$ at $B \gtrsim 20$. The number versus magnitude relation $n(m)$ of $\mathrm{QSOs}^{2}$ is characterized by $\beta \approx 1.0$ at $B \lesssim 18$ and $\beta \approx 0.6$ at $B \gtrsim 18$. The fraction of the sky accessible to the LAMA telescope compared with the fraction of the sky accessible to a conventional telescope are shown in Figure 1, as functions of latitude and maximum zenith angle of the conventional telescope. Assuming a typical latitude of \pm 30 deg for both the LAMA telescope and the conventional telescope and assuming a maximum zenith angle of $60 \mathrm{deg}$ for the conventional telescope, the ratio of the solid angle of the sky accessible to the LAMA telescope to the solid angle of the sky accessible to the conventional telescope is $\Omega_{\mathrm{LAMA}} / \Omega_{\text {other }} \approx 12.5$. Under these assumptions, the decrease of "sensitivity," or the amount by which sources observed by the LAMA telescope will be fainter than the sources observed by a conventional telescope, is summarized in Table 4, for different types of sources.

Table 4

Decrease of "Sensitivity"

\begin{tabular}{|c|c|}
\hline Source & $\overline{\Delta \Delta m}$ \\
\hline Bright $(B \lesssim 20)$ stars .. & 1.6 \\
\hline Faint $(B \gtrsim 20)$ stars & 3.7 \\
\hline Bright $(B \stackrel{\sim}{\prec} 18)$ QSOs & 1.1 \\
\hline Faint $(B \gtrsim 18)$ QSOs & 1.8 \\
\hline
\end{tabular}

For observations of the brightest examples of a class, the decrease of sensitivity of the LAMA telescope in comparison to conventional telescopes ranges from $\Delta m \approx 1.1$ for bright QSOs to $\Delta m \approx 1.6$ for bright stars to larger values for faint stars and QSOs.

\subsection{Summary}

In summary, the implications of the limited pointing and tracking abilities of the LAMA telescope are as follows: 
- All deep observations obtained by the LAMA telescope will contain a temporal dimension as a natural consequence of the design and mode of operation of the telescope. We consider this one of the most important benefits of the telescope.

- For observations of untargeted sources (e.g. faint galaxies and supernovae or faint stars), the LAMA telescope will access a scientifically accessible field of view of more than $10 \mathrm{deg}^{2}$. For observations of targeted sources (e.g. bright QSOs or bright stars), the LAMA telescope will access a scientifically accessible field of view of $\approx 6 \%$ of the entire sky, which is $\approx 10$ times less than the accessible field of view of a conventional telescope pointing to a maximum zenith angle of $60 \mathrm{deg}$. Tens of thousands of QSOs will occur within the scientifically accessible field of view.

- For observations of the faintest instances of a class (e.g. the faintest accessible galaxies and supernovae or the faintest accessible stars), the sources that will be observed by the LAMA telescope will be on average identical to the sources that will be observed by conventional telescopes. For observations of the brightest instances of a class (e.g. the brightest high-redshift QSOs or the brightest nearby stars that harbor extrasolar planets), the sources that will be observed by the LAMA telescope will be on average fainter than the sources that will be observed by conventional telescopes, by an amount that ranges between $\Delta m \approx 1$ and 4 , depending on the type of source.

These results quantify the tradeoffs imposed by the limited pointing and tracking abilities of the LAMA telescope.

\section{SCIENCE OBJECTIVES}

In this section we describe some of the primary scientific objectives of the LAMA telescope project, with particular emphasis on topics related to the scientific interests and background of the authors.

\subsection{LAMA Narrow-Field Survey}

The LAMA telescope will be used to carry out an extremely deep, narrow-field imaging survey at optical and infrared wavelengths in order to identify the first luminous objects in the universe, to identify and study faint, very high redshift galaxies and supernovae, and to establish statistically meaningful samples of very high redshift objects for subsequent observation with second-generation instruments.

Consider 10 fields of high Galactic latitude distributed across the accessible field of view. Each field will be observed for the maximum possible exposure time (i.e. $\approx 30 \mathrm{~min}$ ) at every available opportunity using the multiband optical- and infrared-wavelength camera with incoherent beam combination. Each field will be centered on a suitable phase reference source. Over the course of a year, each field will be observed for a total of $\approx 35$ $\mathrm{hr}$ (assuming $50 \%$ down time due to weather and engineering). The survey will reach limiting specific energy flux densities of $f_{\nu} \approx 0.08 \mathrm{nJy}$ at $\lambda=1 \mu \mathrm{m}$ and $f_{\nu} \approx 0.8 \mathrm{nJy}$ at $\lambda=2.2 \mu \mathrm{m}$, and the survey will cover a solid angle of $\Omega \approx 2.5 \mathrm{arcmin}^{2}$. Over the course of five years, each field will be observed for a total of $\approx 175 \mathrm{hr}$, and the survey will reach limiting specific energy flux densities of $f_{\nu} \approx 0.05 \mathrm{nJy}$ at $\lambda=1 \mu \mathrm{m}$ and $f_{\nu} \approx 0.3 \mathrm{nJy}$ at $\lambda=2.2 \mu \mathrm{m}$.

The LAMA Narrow-Field Survey observations will be used to address the following topics:

- The first luminous objects in the universe: The first luminous objects in the universe were probably massive stars or star clusters, with angular sizes of a few milliarcsec and specific energy fluxes of $\approx 0.1$ to $1 \mathrm{nJy} .^{3}$ The LAMA Narrow-Field Survey observations will identify massive stars and star clusters to redshifts as large as $z \approx 18$.

- Very high redshift galaxies and supernovae: Observations of very high redshift galaxies and supernovae will provide a direct view of the earliest galaxy population. An estimate of the cumulative galaxy surface density versus redshift and magnitude based on measurements of faint galaxies in the Hubble Deep Field is shown in Figure 2. Extrapolations of results of Figure 2 suggest that the LAMA Narrow-Field Survey observations will detect $\approx 20,000$ galaxies, of which $\approx 100$ will be of redshift $z \gtrsim 10$. The LAMA NarrowField Survey observations will also detect supernovae to redshifts $z \gtrsim 10$. Presumably the spectroscopic observations needed to establish masses, chemical abundances, and ionization states of the earliest galaxies will be obtained using the LAMA telescope with second-generation instruments. 

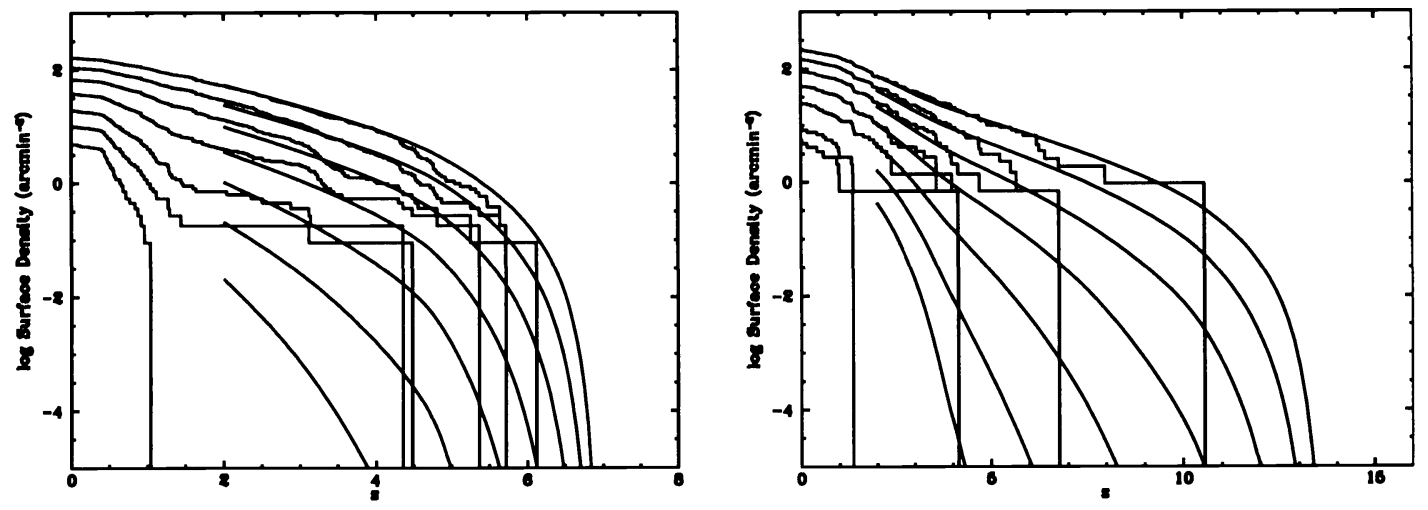

Figure 2. Logarithm of cumulative galaxy surface density versus redshift and magnitude for galaxies selected in the F814W (left panel) and F160W (right panel) bands. Smooth curves are best-fit model, and jagged curves are observations. Different curves show different magnitude thresholds, ranging from $A B=22$ (bottom curves) through $A B=28$ (top curves).

\subsection{LAMA Wide-Field Survey}

The LAMA telescope will be used to carry out a very deep, wide-field imaging survey at optical and infrared wavelengths in order to establish the star formation history of the universe and to probe galaxy evolution over most of cosmic time.

Consider 2000 fields of high Galactic latitude distributed across the accessible field of view. Each field will be observed for the minimum practical exposure time (i.e. $\approx 3 \mathrm{~min}$ ) once every two weeks using the multi-band optical- and infrared-wavelength camera with incoherent beam combination. Each field will be centered on a suitable phase reference source. Over the course of a year, each field will be observed for a total of $\approx 22$ min (assuming 50\% down time due to weather and engineering). The survey will reach limiting specific energy flux densities of $f_{\nu} \approx 0.8 \mathrm{nJy}$ at $1 \mu \mathrm{m}$ and $6 \mathrm{nJy}$ at $2.2 \mu \mathrm{m}$, and the survey will cover a solid angle of 500 arcmin ${ }^{2}$.

The LAMA Wide-Field Survey will be used to address the following topics:

- Star formation history of the universe: Recent observations of the star formation history of the universe ${ }^{4,5}$ suggest that star formation occurred more rapidly in the past. But these observations are incomplete due to selection biases imposed by dust and surface brightness effects. Because the frequency of Type II supernovae is directly related to the star formation rate, the star formation rate history of the universe can be determined without bias from the incidence of Type II supernovae versus redshift. An estimate of the cumulative supernova rate density versus redshift based on measurements of faint galaxies in the Hubble Deep Field is show in Figure 3. Extrapolations of results of Figure 3 suggest that the LAMA Wide-Field Survey observations will identify hundreds of Type II supernovae, at redshifts spanning $z \approx 0$ to 6 .

- Galaxy evolution: Statistically meaningful samples of galaxies at all redshifts $z \approx 0$ to 6 will establish properties of the evolving galaxy population. Extrapolations of results of Figure 2 suggest that the LAMA Wide-Field Survey observations will detect hundreds of thousands of galaxies, of which tens of thousands will be of redshift $z>5$.

\subsection{LAMA Spectroscopic Survey of High-Redshift QSOs}

The LAMA telescope will be used to carry out a high spectral resolution spectroscopic survey of bright, highredshift QSOs in order to study the physical properties of the Ly $\alpha$ forest absorption systems, to directly detect the acceleration of the expansion rate of the universe, to study the chemical enrichment history of the universe, and to search for time and space variations of the physical constants. 

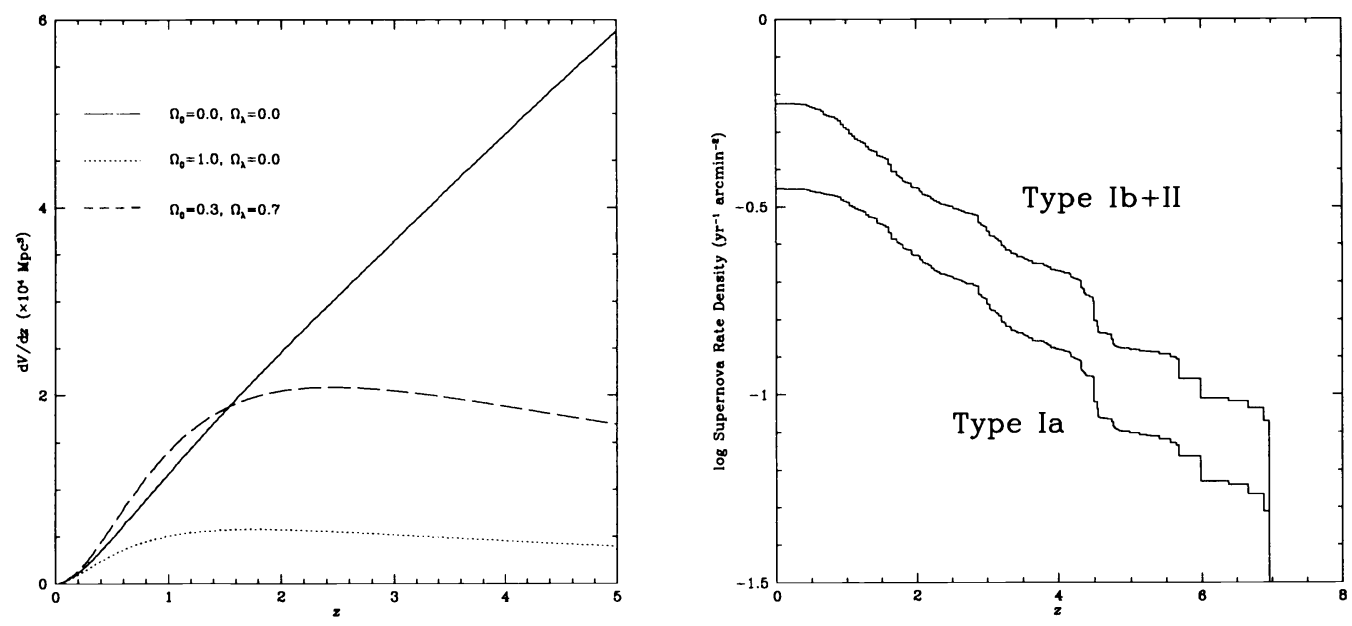

Figure 3. (Left) Differential comoving volume element as a function of redshift for three cosmological models: (1) $\Omega_{M}=0.0$ and $\Omega_{\Lambda}=0.0$, (2) $\Omega_{M}=1.0$ and $\Omega_{\Lambda}=0.0$, and (3) $\Omega_{M}=0.3$ and $\Omega_{\Lambda}=0.7$. (Right) Logarithm of the cumulative supernova rate density for Type Ia and Type Ib+II supernovae at redshifts $0<z<6$ based on measurements of faint galaxies in the Hubble Deep Field.

Consider 100 high-redshift $(z \approx 3-6)$ QSOs distributed across the accessible region of sky. Each QSO will be observed for the maximum possible exposure time (i.e. $\approx 30 \mathrm{~min}$ ) about 18 times per year using the highdispersion $(R \approx 100,000)$ echelle spectrograph. The QSOs will be bright enough $(I \approx 18)$ to serve as reference sources for low-order adaptive optics correction. Over the course of five years, each QSO will be observed for a total of $\approx 22.5 \mathrm{hr}$ (assuming $50 \%$ down time due to weather and engineering). The survey will produce opticalwavelength spectra of velocity resolution FWHM $\approx 3 \mathrm{~km} \mathrm{~s}^{-1}$ and signal-to-noise ratio $S / N \approx 500$ per resolution element.

The Spectroscopic survey of high-redshift QSOs observations will be used to address the following topics:

- Physical properties of the Ly $\alpha$ forest: Physical properties of the Ly $\alpha$-forest absorption systems probe the chemical abundances of early, near-primordial material, the history of the far-ultraviolet radiation field produced by the first sources of ionizing photons, and the epoch of the reionization of the universe. The LAMA QSO Survey will provide observations that bear directly on all of these issues.

- Direct detection of acceleration of the expansion rate: The redshifts of cosmic sources change with time due to (1) acceleration of the expansion rate of the universe and (2) peculiar accelerations caused by local mass concentrations along the line of sight. The accelerations due to both effects is of magnitude $\sim 1 \mathrm{~m}$ $\mathrm{s}^{-1}$ century $^{-1}$. The redshift dependence of the acceleration of the expansion rate of the universe is shown in Figure 4, for different choices of the equation of state of the universe. Simulations of the proposed observations suggest that direct detection of these effects is within reach of the LAMA QSO Survey, if systematic measurement errors can be controlled or accounted for.

- Cosmic chemical evolution: The growth of the chemical content of galaxies with time is linked to many issues of galaxy evolution, including the star formation history, stellar initial mass function, feedback from supernova energy input, and infall of primordial material. The highest column density QSO absorption systems directly probe this very epoch, although measurements of accurate gas column densities (and hence element abundances) is always difficult due to unresolved velocity structure of highly saturated absorption lines. The LAMA QSO Survey observations will provide the spectral resolution and signal-to-noise ratios needed to measure accurate gas column densities. 


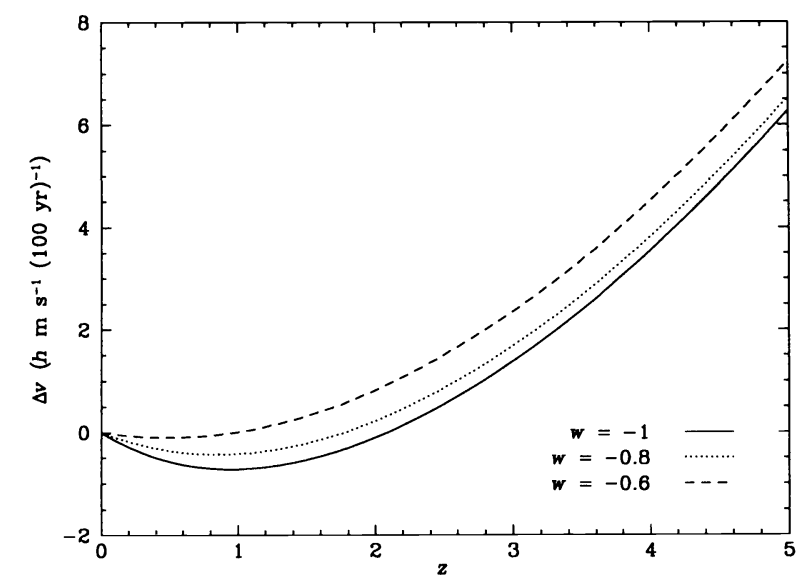

Figure 4. Redshift dependence of the acceleration of the expansion rate of the universe, for three different choices of the equation of state of the universe.

- Time and space variations of the physical constants: Recent observations ${ }^{6,7}$ have tentatively detected temporal variation of the fine structure constant, at a level of $\Delta \alpha / \alpha=-0.57 \pm 0.10 \times 10^{-5}$ averaged over the redshift interval $z=0.2-3.7$. The LAMA QSO Survey will measure variation of the physical constants versus time and space, thereby confirming or refuting present results and possibly tracing variation of the physical constants versus time and space.

\section{SUMMARY AND CONCLUSIONS}

The LAMA telescope is unconventional in both design and method of operation. Although the LAMA telescope will not have all of the capabilities of a conventional large-aperture telescope-due primarily to the constraint of near-zenith pointing and tracking -it will be used to address important issues in cosmology and extragalactic and galactic astronomy.

\section{ACKNOWLEDGMENTS}

The authors gratefully acknowledge the contributions of the LAMA scientific team, including Hsiao-Wen Chen, Arlin Crotts, Aaron Evans, David Helfand, Mike Shara, Fred Walter, and John Webb. Development of the LAMA telescope project has been made possible by generous financial support provided by Stony Brook University and Columbia University.

\section{REFERENCES}

1. Infante, L. 1994, A\&A Supp, 107, 413

2. Crampton, Cowley, \& Hartwick 1987

3. Miralda-Escudé, J., \& Rees, M. 1998, ApJ, 497, 21

4. Madau, P. et al. 1996, MNRAS, 283, 1388

5. Lanzetta, K. M., et al. 2002, ApJ, 570, 492

6. Webb et al. 2001, Phys. Rev. Lett. 87, 91301

7. Murphy et al. 2001a, MNRAS, 327, 1237 